\title{
Lubricin-inspired Loop Zwitterionic Peptide for Fabrication of Superior Antifouling Surfaces
}

Chuanxi Li, ${ }^{a, d, \perp}$ Yinqiang Xia ${ }^{b, \perp}$ Chunjiang Liu, ${ }^{a,{ }^{*}}$ Renliang Huang, ${ }^{c}$ Wei Qi, ${ }^{a}$ Zhimin He, ${ }^{a}$ and Rongxin Sua,c,*

a State Key Laboratory of Chemical Engineering, Tianjin Key Laboratory of Membrane Science and Desalination Technology, School of Chemical Engineering and Technology, Tianjin University, Tianjin 300072, PR China

${ }^{\mathrm{b}}$ College of Food Science and Engineering, Northwest A\&F University, Yangling, Shaanxi 712100, PR China

c School of Marine Science and Technology, Tianjin University, Tianjin 300072, PR China d Petrochemical Research Institute, PetroChina, Beijing 102206, PR China

${ }^{\perp}$ C.L. and Y.X. contributed equally to this work.

* Author to whom any correspondence should be addressed:

E-mail: surx@tju.edu.cn (R. S.); ; cjliu@tju.edu.cn (C. L.) 


\section{Supplementary Figures}
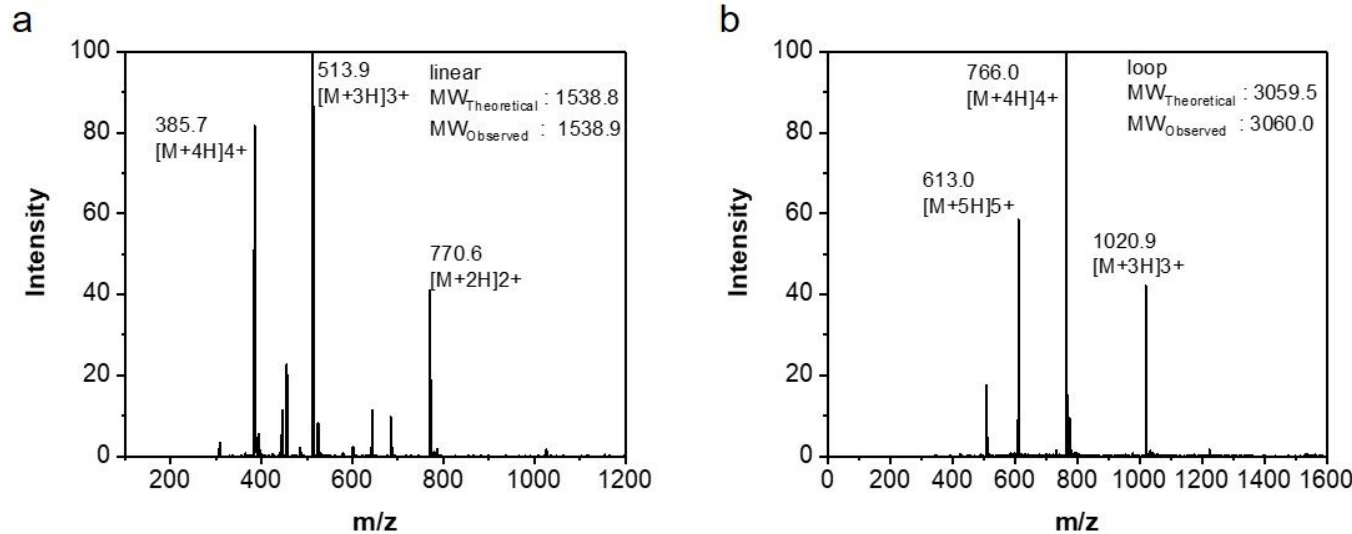

Figure S1. The mass spectrum of linear-like (a) and loop-like (b) zwitterionic peptide.

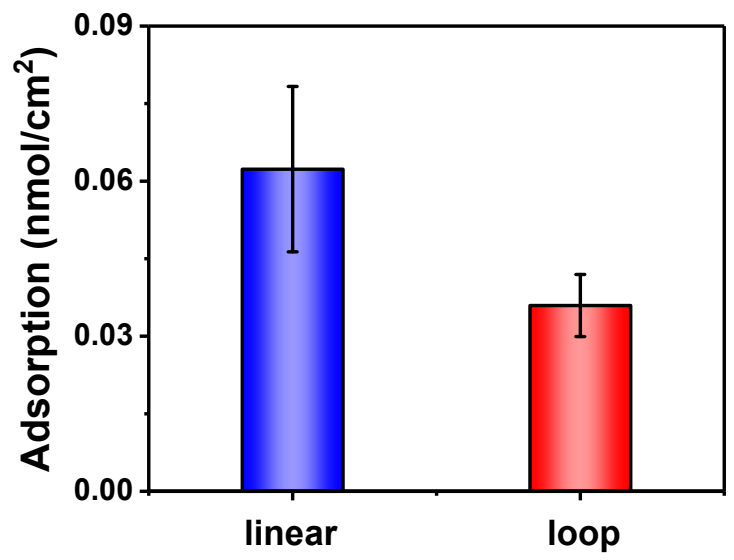

Figure S2. The modification quantities of zwitterionic peptide with linear or loop structure.

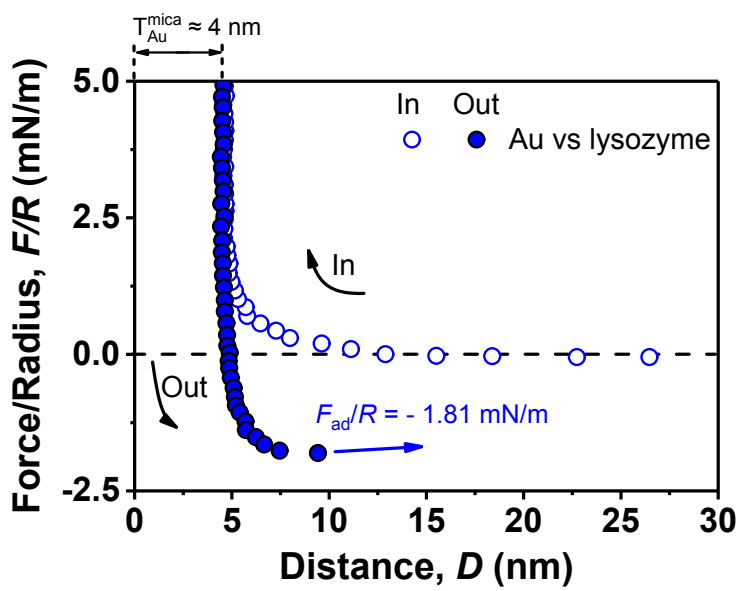

Figure S3. SFA force curves between lysozyme and a bare gold surface. 

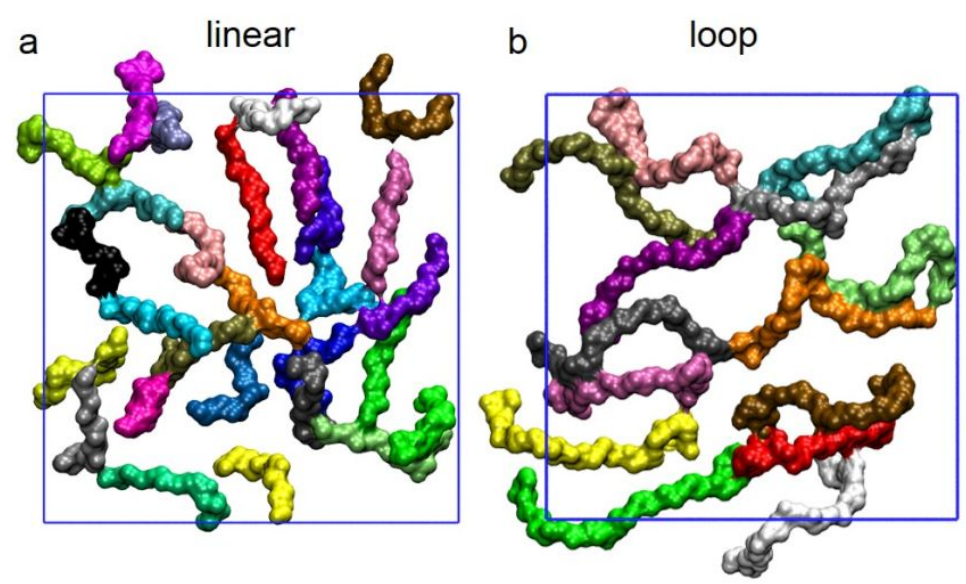

Figure S4. The snapshot of linear-like (a) and loop-like (b) zwitterionic peptides. Chains are differentiated by color.

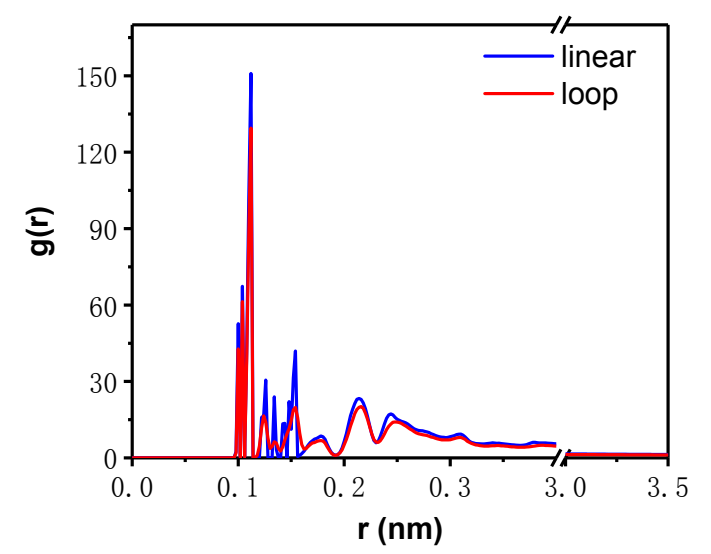

Figure S5. The radial distribution function of linear or loop zwitterionic peptide chains around themselves. Higher peaks of $g(r)_{\text {linear }}$ around $0.1-0.15 \mathrm{~nm}$ indicate there are more chains around themselves.

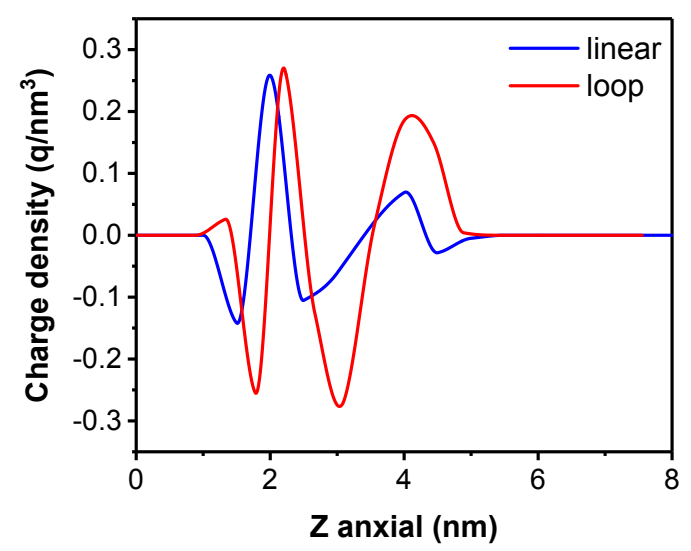

Figure S6. The charge distribution of lineal/loop-like zwitterionic peptide-modified surface. 


\section{Supplementary Tables}

Table S1. The p-values of the t-test analysis of the antifouling performance for BSA.

\begin{tabular}{|c|ccc|}
\hline & bare & loop & linear \\
\hline bare & $/$ & $2.84 \mathrm{E}-05$ & $3.26 \mathrm{E}-05$ \\
loop & $/$ & $/$ & 0.092 \\
linear & $/$ & $/$ & $/$ \\
\hline
\end{tabular}

Table S2. The p-values of the t-test analysis of the antifouling performance for lysozyme.

\begin{tabular}{|c|ccc|}
\hline & bare & loop & linear \\
\hline bare & $/$ & $6.16 \mathrm{E}-05$ & $7.05 \mathrm{E}-05$ \\
loop & $/$ & $/$ & 0.023 \\
linear & $/$ & $/$ & $/$ \\
\hline
\end{tabular}

Table S3. The p-values of the t-test analysis of the anti-bacterial assay.

\begin{tabular}{|c|ccc|}
\hline & bare & loop & linear \\
\hline bare & $/$ & $9.60 \mathrm{E}-06$ & $1.46 \mathrm{E}-05$ \\
loop & $/$ & $/$ & 0.010 \\
linear & $/$ & $/$ & $/$ \\
\hline
\end{tabular}

